Michael R. Ott

\title{
Black Box Buchdruck
}

\section{Geheimhalten und Verbergen}

Es ist hinlänglich bekannt und wird auch immer wieder aufgeschrieben, dass unsere Kenntnisse über die Anfänge des Drucks mit beweglichen Lettern begrenzt sind. Zwar wissen wir das ein oder andere, aber wir wissen nicht so viel, wie wir angesichts der beträchtlichen Auswirkungen dieser „Prozessinnovation“1 gerne wüssten. Diese Enttäuschung, dieses enttäuschte Wissensbegehren, betraf in der Vergangenheit insbesondere auch nationalistisch orientierte Bemühungen, die darauf abzielten, den Druck mit beweglichen Lettern zu einer genuin deutschen Erfindung zu machen. Dieses Wissensbegehren lässt sich offenbar nicht in gewünschtem Maße erfüllen.

Das, was wir über den Druck mit beweglichen Lettern wissen, wissen wir - erstens - aus Rekonstruktionen anhand späterer Apparate, auch aus späteren Aufzeichnungen sowie aus späteren Abbildungen; was wir wissen, wissen wir - zweitens - aus Gerichtsakten, in denen Probleme und Konflikte der technischen Großunternehmen des frühen Letterndrucks punktuell sichtbar werden; und drittens wissen wir - zumal die frühen Apparate nicht mehr vorhanden sind - einiges aufgrund der Produkte des Drucks mit beweglichen Lettern, also aufgrund derjenigen gedruckten Artefakte, die bis heute erhalten geblieben sind und aus denen wir Rückschlüsse auf Techniken, Diskurse und Praktiken ziehen können.

Abgesehen von oftmals überzogenen Erwartungen an Zeugnisse einer Innovation, deren Effekte zum Innovationszeitpunkt ja noch gar nicht abzusehen waren, mag der oft beklagte Mangel an Informationen über den frühen Letterndruck mit der recht banalen Tatsache zu tun haben, dass Aufzeichnungen und Skizzen zu den technischen Experimenten, zu den Werkzeugen, Maschinen und Abläufen über die Jahrhunderte hin verloren gingen - so wie zahllose andere Dokumente eben auch. ${ }^{2}$ Doch selbst wenn es durchaus möglich ist, dass während der langen Experimentierphase zu Beginn des Drucks mit beweglichen Lettern - quasi als Laborbuch - Aufzeichnungen angefertigt wurden, wäre deren Verschwinden wohl nicht einfach nur ein bedauerlicher Zufall, sondern auch ein Effekt des transitorischen Status solcher Notizen, die immer nur gebraucht werden, bis ein gegebenes Problem zufriedenstellend gelöst ist.

1 Zinn 1989, 34.

2 Zum heutigen (Nicht-)Wissen über Gutenberg siehe etwa Wagner 2000.

Dieser Beitrag ist im Heidelberger Sonderforschungsbereich 933 „Materiale Textkulturen. Materialität und Präsenz des Geschriebenen in non-typographischen Gesellschaften“ entstanden (Teilprojekt C05 „Inschriftlichkeit. Reflexionen materialer Textkultur in der Literatur des 12. bis 17. Jahrhunderts“). Der SFB 933 wird durch die Deutsche Forschungsgemeinschaft finanziert. 


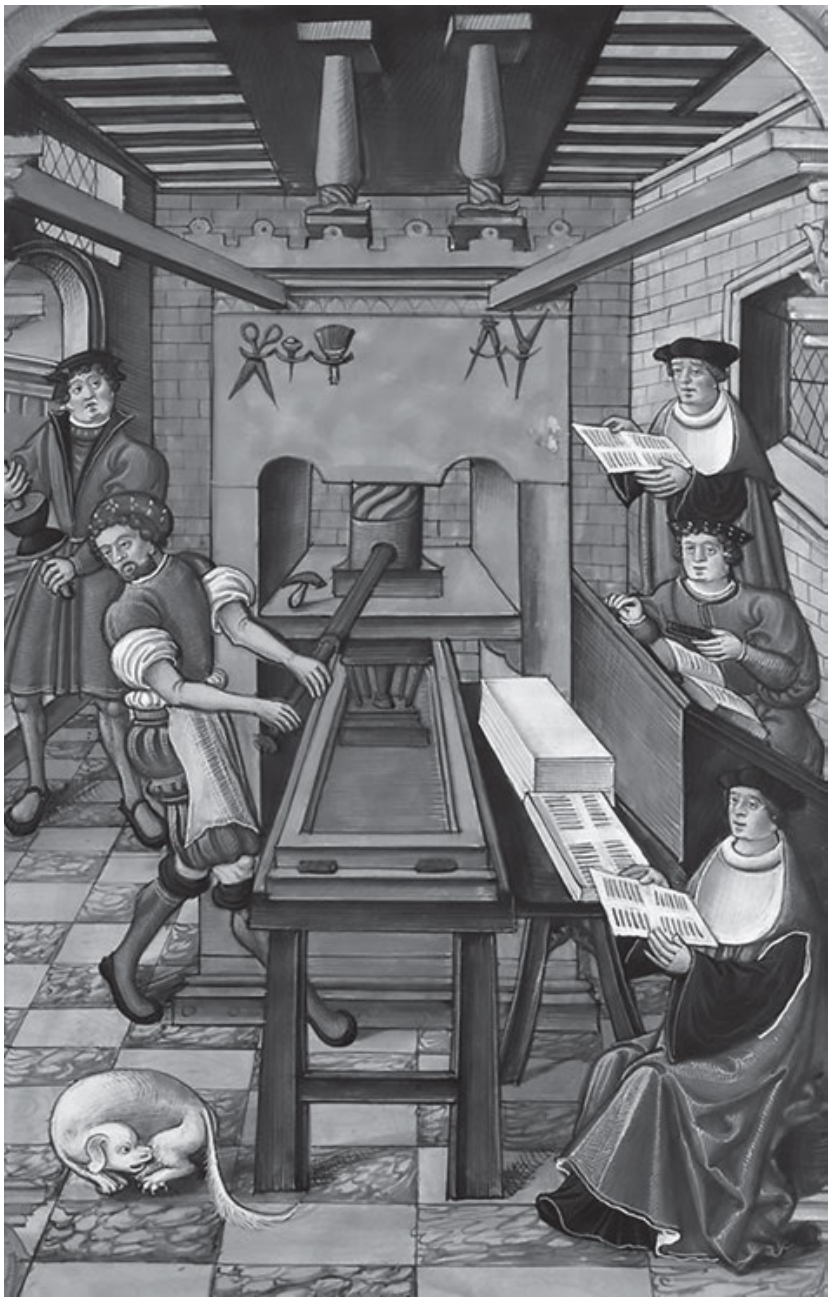

Abb. 1:

Eine Druckerwerkstatt in einer Darstellung des frühen 16.Jahrhunderts.

So gesehen wäre die Opazität des frühen Letterndrucks nicht nur das Ergebnis verlorener Dokumente, sondern ebenso ein Effekt der technischen Innovation im Laborbetrieb. Zudem gab es im 15. Jahrhundert aber auch wenig Grund und wenig Notwendigkeit, die Gestaltung des „Typographeums“,3 die darin verrichteten Tätigkeiten und das dafür notwendige Wissen zu kodifizieren; gerade handwerkliche Tätigkeiten wurden in aller Regel nicht aus Büchern, sondern qua Praxis gelernt und gelehrt, also durch die räumliche Nähe von erfahrenen und (noch) unerfahrenen Personen sowie durch die gemeinsame Produktion. So gesehen wäre die Opazität des frühen Letterndrucks auch ein Effekt der Art und Weise, wie handwerklich-technisches Wissen weitergegeben wird.

3 Giesecke 22017 [1991]. 
Aus der Frühzeit der Prozessinnovation, die ich im Folgenden meist grob verkürzt „Buchdruck“ nenne, ist also wenig aus dem bekannt, was HistorikerInnen „Quellen“ nennen. ${ }^{4}$ Dies hat neben den eben genannten Gründen zwei weitere Ursachen und beide haben etwas damit zu tun, dass der Buchdruck von Anfang an und mit massivem Aufwand als Black Box konzipiert wurde: Gutenbergs Werkstatt (und nur um die wird es mir gehen) nimmt Material auf („Input“) und stößt Geschriebenes aus („Output“); was aber dazwischen passiert, das bleibt den Blicken weitgehend entzogen und soll auch verborgen bleiben. Das, was den Blicken entzogen wird und opak bleibt, lässt sich im Rahmen einer historischen Black Box-Analyse zwar durchaus erhellen; mir aber - und das ist ein Vorteil der Perspektive, die man einnimmt, wenn man über Black Boxes nachdenkt -; mir aber geht es darum, zum einen die Ursachen und Effekte zu beschreiben, die mit der Black Box Buchdruck einhergehen und zum anderen den langen Linien zu folgen, die sich von der Black Box Buchdruck bis hin zu heutigen Textverarbeitungskonstellationen ziehen lassen.

Was nun das ,Black Boxing ${ }^{65}$ des frühen Buchdrucks anbelangt, so wird dies vor allem anhand von zwei Aspekten deutlich. Ein Aspekt betrifft die Produktion, der andere die Rezeption. Zum einen nämlich war der Unternehmer Johannes Gensfleisch, den man auch Gutenberg nannte, gezwungen, sein ,Labor` (so wie später seine Werkstatt) vor neugierig-interessierten Blicken möglichst zu schützen, um von technischen Innovationen längerfristig profitieren zu können. „Es lohnte sich für die ersten Druckergenerationen offenbar, ihr Fachwissen geheimzuhalten“, schreibt Michael Giesecke in seinem vielgelesenen Standardwerk aus dem Jahr 1991. ${ }^{6}$ Ein solches Bedürfnis nach Geheimhaltung lässt sich in Gutenbergs Straßburger Zeit etwa für seinen ersten nachweisbaren Wohnsitz vermuten:

1434 wohnte Gutenberg im Bereich des umfriedeten Klosters St. Arbogast, 2,5 km außerhalb der Straßburger Stadtmauern. Gleich an den Ufern der Ill gelegen, bot dieser Standort gewisse Vorteile für Gutenbergs Aktivitäten. Hier war er relativ unbehelligt und vor allen Dingen geschützt vor allzu neugierigen Augen und konnte ungestört seine technischen Versuche durchführen. Eine solche Geheimhaltung war in einem Jahrhundert unerlässlich, in dem es keinen Patentschutz oder andere rechtliche Absicherung zur Wahrung einer innovativen Idee gab. Darüber hinaus bot sich die Ill als Antriebsquelle für Mühlräder, Hammerwerke oder ähnlich schwere technische Hilfsmittel an. ${ }^{7}$

Die Vermutungen, die Sabina Wagner hier anstellt, sind plausibel, weil es sich bei Gutenberg um einen Unternehmer handelt, der an technischen Innovationen interessiert war und deshalb einen Ort brauchte, der einen Labor- und Werkstattbetrieb

4 Die wichtigsten Zeugnisse zu Gutenberg sind abgedruckt und erläutert bei Schorbach 1900.

5 Mehr zu dieser Praxis (im Unterschied zur Black Box als Artefakt) im Aufsatz von Christoph Borbach in diesem Band.

6 Giesecke 22017 [1991], 69.

7 Wagner 2000, 123b. 
ermöglichte; einen Ort, der vor neugieriger Beobachtung - mithin vor frühen Formen von Industriespionage - gut geschützt war.

In schriftlichen Zeugnissen fassbar wird eine Betriebsamkeit in Gutenbergs Straßburger Laborwerkstatt für das Jahr 1438, als dort, offenbar in großer Menge, Tragezeichen für eine Wallfahrt nach Aachen im Folgejahr produziert wurden. Vor dem Hintergrund der späteren Innovation in Sachen Letterndruck ist die Produktion der Tragezeichen wohl ein Schritt in Richtung mechanischer und massenhafter Vervielfältigung. So gesehen ist die Werkstatt, in der die Tragezeichen produziert wurden, aus Buchdruckperspektive zugleich ein Labor, in dem wichtige und innovative Entwicklungen stattfanden. Deshalb gilt es, die Arbeit in diesem Labor geheimzuhalten.

Beim Black Boxing des frühen Buchdrucks geht es also auch um ,wirtschaftliche“ Gründe. Die technischen Innovationen, die für eine Massenproduktion von Tragezeichen notwendig waren, sind monetarisierbar, wenn und insofern diese Innovationen gezielt und gegen Gegenleistung weitergegeben werden. Zwar lässt sich die Ausbreitung technischer Innovation angesichts einer begehbaren Black Box wie derjenigen Gutenbergs natürlich nicht verhindern (immerhin sind Menschen in der Produktion beteiligt), ${ }^{8}$ aber zum einen kann man darauf achten, aus der Ausbreitung Kapital zu schlagen und zum anderen kann man durch kontinuierliche Innovation versuchen, geldwertes Wissen kontinuierlich vorzuhalten, indem man sich bemüht, der Konkurrenz stets mindestens einen Schritt voraus zu sein. Auf jeden Fall verwundert es nicht, dass der Druck mit beweglichen Lettern in der Frühphase anhand und entlang von Menschen verbreitet wurde, die bereits in Buchdruckwerkstätten gearbeitet hatten. Nur diese Akteure verfügten über ausreichend Wissen, um die Gutenbergsche Werkstatt zu reproduzieren.

Dies ist der eine Grund, warum der frühe Druck mit beweglichen Lettern von Anfang an als Black Box konzipiert war. Zum anderen war der Buchdruck in seinen Anfängen darauf ausgelegt, sich im Endprodukt unsichtbar zu machen. Hier wie in vielen anderen Fällen imitiert das neue Medium das alte (,Skeuomorphismus') und das fertige Produkt verbirgt sehr effektiv die Aufwände, die es überhaupt ermöglicht haben. Mit Gutenbergs Bibel - von Anfang an als proof of concept konzipiert - wurde denn auch allem Anschein nach nicht das Ziel verfolgt, etwas herzustellen, was anders als Handschriften aussah. Vielmehr hatte man das Ziel, etwas zu produzieren, das wie eine perfekte Handschrift wirkte. Erst die perfekte und perfektionierende Imitation von Handschriften konnte den Druck mit beweglichen Lettern anschlussfähig machen an die Handschriftenkultur. Alle weiteren ,Vorteile‘ des Buchdrucks (Ähnlichkeit, hohe Anzahl, niedrigerer Preis) sind wichtig, aber gegenüber der Anschlussfähigkeit an die Handschriftenkultur vorerst zweitrangig. Zuerst steht die Aufgabe an,

8 Von Druckern der zweiten Generation, nämlich von Johannes Fust und Peter Schöffer, wissen wir, dass sie die Arbeiter durch Schwur zur Geheimhaltung verpflichtet haben - was natürlich nicht lange funktioniert hat. Näheres dazu bei Giesecke 22017 [1991], 211. 
den riesigen Aufwand unsichtbar zu machen, der notwendig ist, um in einer Werkstatt im Druckverfahren perfekte Handschriften zu produzieren. Man stellte sich also auf die Erwartung der ,NutzerInnen“ ein und schafft ein Produkt, dessen ,Nutzeroberfläche“ bisherige Sehgewohnheiten nur insofern irritiert, als es scheinbar von hoher mensch-manueller Qualität ist (obwohl es ein mensch-maschinelles Produkt ist).

Angesichts der manuskriptähnlichen Produkte verwundert es nicht, dass man kaum jemanden findet, der sich im 15. Jahrhundert kritisch zum Buchdruck selbst geäußert hat. Kritisiert wird mitunter die moralische Eignung der Drucker, nicht aber die technische Innovation und deren Erzeugnisse. ${ }^{9}$ Erst Schritt für Schritt - und selbstverständlich nicht im Rahmen einer geradlinigen Entwicklung - wird man sich in den folgenden Jahrzehnten von dem Vorbild der Handschrift lösen und also das Schriftbild vereinfachen sowie neue Buchelemente (wie zum Beispiel die Titelblätter) entwickeln. Parallel dazu wird dann auch der Buchdruck zu einem normalen Handwerk, das wiederum, etwa im Fall des Schriftgießers, auf verschieden Handwerke zurückgreift. Über die technischen Details des Buchdrucks kann man sich dann vielerorts ohne größeren Aufwand informieren.

Diese beiden Gründe für das Black Boxing des Buchdrucks - das Verheimlichen und das Verbergen - sind in der Forschung weithin bekannt und so lohnt es eigentlich nicht, sie noch einmal aufzuschreiben. Dass ich es trotzdem tue, hängt vor allem damit zusammen, dass ich mir ansehen möchte, wie die Black Box Buchdruck im Anschluss an Gutenberg (wiederholt) geöffnet, geschlossen und schließlich ins Desktop Publishing ,übersetzt' wurde. Auf diese Weise nämlich lässt sich vielleicht zeigen, wie die Elemente in dem schwarzen Kasten - all die menschlichen und nicht-menschlichen Akteure, all die Maschinen, all die Prozesse, all die Arbeit - verschiedentlich neu arrangiert und neu verpackt wurden. Dabei komme ich zum Schluss, dass die Computerisierung der Buchproduktion dazu geführt hat, dass zahlreiche Bestandteile der ursprünglichen Black Box heute hineinverlagert wurden in ein hochkomplexes, dichtes und mächtiges Maschinen-Mensch-Arrangement; hineinverlagert in Akteure, die Aufgaben übernehmen, die zuvor AutorInnen, SetzerInnen, KorrektorInnen, DruckerInnen und BuchhändlerInnen inne hatten.

Das Mensch-Maschinen-Arrangement des frühen Buchdrucks ist also über die Jahrhunderte vielfach transformiert worden und so kann man sich schließlich auch fragen, was das eigentlich ist, das die Black Box Buchdruck heute produziert. Solche Bücher, wie sie die Black Box 500 Jahre lang verfertigt hat, gibt es heute immer seltener. Was aber produziert man heute? Sind es Texte, Zeichenketten, Schriftströme? Wie sieht es aus, das Aufschreibesystem 2000, ${ }^{10}$ über das Friedrich Kittler in seiner immer auch zur Germanistik zählenden Habilitationsschrift schon deshalb nicht mehr hat schreiben können, weil es nicht mehr zum Literaturdispositiv gehört? Vielleicht kann

9 Eisenstein 2011, 7.

10 Kittler ${ }^{4} 2003$ [1985]. 
man sagen, dass das Aufschreibesystem 2000 geprägt ist durch permanente Potentialität, durch noch nicht ausgeschöpfte Möglichkeiten der produzierten Texte, durch Entgrenzung und Unabgeschlossenheit. Aus der Sicht eines Mediävisten heißt dies auch - und dies ist ohnehin eines der Lieblingsargumente von MediävistInnen -, dass der Druck mit beweglichen Lettern eine rund fünfhundertjährige Phase der Stillstellung des Geschriebenen markiert und dass die digitale Textkultur ein wenig an die Beweglichkeit der Handschriftenkultur, an deren mouvance und deren variance erinnert. ${ }^{11}$

\title{
2 Mensch und Maschine
}

Gedruckte Bücher gab es vor dem Druck mit beweglichen Lettern, zumal die grundlegende Idee der Wiederverwendung einer einfärbbaren Vorlage hinreichend schlicht ist, gerade im Vergleich zu denjenigen Werkstätten, die dann seit der Mitte des 15. Jahrhunderts notwendig sind, um Gedrucktes mit beweglichen Lettern zu produzieren. Die konkreten Abläufe und Aufwände in einer solchen typographischen Werkstatt sind der heutigen Forschung mittlerweile einigermaßen klar, weil man die Black Box seit dem 19. Jahrhundert geöffnet und also von späteren Artefakten, Berichten und Abbildungen auf Gutenbergs Werkstatt rückgeschlossen hat (und aus Mangel an anderen Zeugnissen auch rückschließen muss) und weil man durch experimentelle Archäologie die Herausforderungen und Tätigkeiten einigermaßen rekonstruieren kann. ${ }^{12}$ Die Aufgaben, die Gutenbergs Labor zu lösen hatte, bevor es zur Werkstatt werden konnte, waren, so lässt sich heute sagen, vielfältig:

\begin{abstract}
Um diese kleinen Werke mit beweglichen Lettern herzustellen, hatte es zahlloser Versuche und Einzelerfindungen bedurft, die vor allem sehr viel Zeit und Geld kosteten. Diese begannen mit dem Entwurf der Schrift, dem Gravieren der Patrize, der Herstellung der Matrizen, der Erfindung des Handgießgeräts und der Zusammensetzung der Metalllegierung, der Anpassung der Buchstaben für einen geraden Zeilenstand, dem Finden der richtigen Rezeptur für die Druckfarbe und reichten bis zu den Experimenten mit Papier und Pergament, die für die ideale Aufnahme der Druckfarbe vorbereitet sein mussten. Versuche mit der Presse und ihrer Mechanik waren notwendig, um zahllose kleine Details auszuarbeiten. Bis zur Satzgestaltung und der richtigen Setztechnik musste vieles neu erdacht, gefunden und erprobt werden. ${ }^{13}$
\end{abstract}

Je näher man hinsieht, desto komplexer werden selbst kleinere, beständig wiederkehrende Arbeitsschritte - und allein die Beobachtung solcher Arbeitsschritte reicht mitunter nicht aus, um das Praxiswissen zu erschließen, das sich hinter den

11 Grundlegend zur Beweglichkeit der Textualität von Handschriftenkulturen Cerquiglini 1989.

12 Man vergleiche Hanebutt-Benz 2000a, 158.

13 Wagner 2000, $130^{\mathrm{a}}$. 
routinisierten Tätigkeiten verbirgt und das mitunter nicht-expliziertes Körperwissen ist. Ein Beispiel sind die Druckerballen, mit deren Hilfe Farbe vom Farbtisch aufgenommen wurde, um dann „mit gleichmäßigen, kreisenden Bewegungen“ die Oberfläche des Satzes mit Farbe zu versehen:

Der Ballen selbst bestand aus einem hölzernen gedrechselten Griff und dem Ballholz (leichtes und zähes Holz wie Linde, Erle oder Ahorn), einer schüsselförmigen Scheibe, die Innenseite leicht konkav gestaltet, um möglichst viel Füllung aufnehmen zu können. Das Füllmaterial war Wolle oder Rosshaar. Der Durchmesser betrug ca. $20 \mathrm{~cm}$. Als Bezugsmaterial werden Schafsleder, Kalbsleder und Hundeleder genannt. Das Leder wurde kreisförmig zugeschnitten, nachdem es gut präpariert war. Es durfte nicht fettig sein, um die Druckerfarbe gut annehmen zu können. So wurde es in Bier getaucht, gewalkt bzw. mit den Händen gerieben und ausgewrungen oder über Nacht in Wasser eingeweicht, am nächsten Tag so lange mit den Füßen bearbeitet, bis das Leder ganz weich war, dann von überschüssiger Feuchtigkeit befreit, nach dem Trocknen noch auf der Außenseite mit Öl übergossen und nochmals gewalkt. [...] Um aber immer ,gute‘ Ballen zu haben, musste man sie alle drei Tage abbrechen, d. h. sie an drei Falzen öffnen, die Haare herausnehmen und sie zausen (auseinander ziehen), und sie mit trockenen und gut gezausten Haaren, die vorrätig zu sein hatten, neu stopfen und zunageln. ${ }^{14}$

Diese Beschreibung ist, gerade wegen ihrer Liebe zum Detail, ein anschauliches Beispiel dafür, wie in der Forschung die Black Box Buchdruck geöffnet und wie damit ein Blick in die Werkstatt ermöglicht wurde, ganz so wie die Techniksoziologie Blicke in Labore ermöglicht hat, die eigentlich eine abgeschlossene Sphäre darstellen. Eine solche quasi techniksoziologische Forschung ist auch hinsichtlich des Buchdrucks notwendig, weil wir vom Druck mit beweglichen Lettern seit gut 100 Jahren (seit der Technisierung des Drucks durch Rotationspresse und Offset-Druck) zu weit entfernt sind, als dass Wissen über diese Werkstätten gesellschaftlich vorhanden und also einer Nachforschung grundsätzlich zugänglich wäre. Außerdem - und das wiegt wohl schwerer als das fehlende Wissen -; außerdem ist ,uns heute, wo wir von Kind an mit den Erzeugnissen dieser Technologie umgehen, der Sinn für die Komplexität weitgehend abhanden gekommen“", ${ }^{15}$ zumal die heutigen Druckartefakte mit dem frühen Buchdruck nur entfernt etwas zu tun haben. Die Buchakteure von heute haben es mit einem hochgradig standardisierten industriellen Massenprodukt $\mathrm{zu}$ tun. Und dieses Massenprodukt namens Buch verbirgt effektiv die Technikgeschichte, durch die es überhaupt erst möglich geworden ist.

Die Spezialisierung, Automatisierung und schließlich Digitalisierung des Buchdrucks seit der Zeit um 1900 hat denn auch im 20. Jahrhundert zu einer Transformation geführt, die kaum mehr Beziehungen aufweist zu den ,alten` Werkstätten, die gemeinhin ,Offizin' genannt werden. Eine Offizin ist eine komplexe Werkstatt zur Produktion komplexer Waren und sie ist der Schauplatz einer Interaktion von

14 Hanebutt-Benz 2000, $177^{\mathrm{a}}-178^{\mathrm{a}}$.

15 Giesecke 22017 [1991], 68. 
Autor, Verleger, Drucker und Buchhändler sowie der Ort, an dem sich das gedruckte Buch als (ein mögliches) Endprodukt im Zuge vielfältiger Arbeitsschritte und Rückkopplungsschleifen manifestiert. Diese Arbeitsschritte und Rückkopplungsschleifen reichen von der Abgabe des Manuskripts, der Bearbeitung des Manuskripts für den Satz, der Korrektur des Satzes bis hin zum korrekten Arrangement der bedruckten Lagen. ${ }^{16}$

Spätestens im 20. Jahrhundert war von dieser gemeinsamen Arbeit vor Ort nicht mehr viel übrig. Arbeitsteilung und Dezentrierung haben zur räumlichen Trennung von AutorInnen, VerlegerInnen, DruckerInnen und BuchhändlerInnen geführt. Seit dem 21. Jahrhundert allerdings löst sich die Arbeitsteilung mehr und mehr auf und die verschiedenen, zuvor spezialisierten Tätigkeiten fallen in einzelnen Akteuren zusammen: Heutzutage setzen wir mehr und mehr selbst, wir korrigieren selbst, wir produzieren selbst, wir veröffentlichen selbst und wenn wir die Dokumente, die wir produzieren, online stellen, dann zahlen wir dafür nichts. In unseren posttypographischen Zeiten geht es eben gerade nicht um Buchproduktion, sondern, kurz gesagt, um „Textverarbeiten“. ${ }^{17}$ Das ist das Wort, das der IBM-Manager Ulrich Steinhilper um das Jahr 1950 herum ersann, um - in Anlehnung an die gerade modern gewordene „Datenverarbeitung“ - das Schreibmaschinengeschäft der International Business $M a$ chines Corporation begrifflich aufzuwerten. Während in einer ersten Phase der Begriff „eine Frage der Arbeitsorganisation“ bezeichnete, „eine Angelegenheit von Sekretärinnen, Sachbearbeitern und Vorgesetzten sowie den ihnen zur Verfügung stehenden Geräten, um das Schreiben im Büro möglichst effizient zu gestalten“; während also der Begriff in einer ersten Phase die büroliche Arbeitsorganisation meinte, „zog“ mit dem Aufkommen des PC „Textverarbeitung in den privaten Raum ein“ und „der heimische Schreibtisch [wurde] zum Ort des Textverarbeitens““. ${ }^{18}$ Und das hatte Folgen:

Gegenwärtig bedeutet Textverarbeiten somit, die Rolle des Schreibers mit denen des Lektors/Korrektors und des Typografen zusammenzubringen und vormals getrennte Phasen der Dokumentenerstellung (Texte entwerfen, gliedern, skizzieren, formulieren, überarbeiten, korrigieren, formatieren, layoutieren und drucken) auf einer technischen Plattform - dem PC - zu integrieren. ${ }^{19}$

Auf diese Weise tendiert die aktuelle Entwicklung interessanterweise dazu, die Spezialisierung aufzuheben, die auf die Offizin folgte. Am Ende dieser Despezialisierung steht eine Inkorporation der Offizin: Mit Hilfe eines mit dem Internet verbundenen Computers können einzelne Akteure all die Aufgaben übernehmen, die im historischen Rückblick zuerst an einem Ort und anschließend von verschiedenen Institutionen und ExpertInnen übernommen worden waren. Der PC mag anfänglich eine

16 Detaillierte Prozessdiagramme bei Giesecke 22017 [1991], 110, 116.

17 Heilmann 2015. Man lese auch (vom gleichen Verfasser) Heilmann 2012.

18 Heilmann 2015, 586f.

19 Heilmann 2015, 591. 
,bessere Schreibmaschine‘ gewesen sein; aber das war nur der Anfang. Die computerisierte Arbeitsumgebung ermöglicht es, möglichst viele Aspekte des Buchdrucks wieder an einem Ort zu versammeln, so wie einst in der Offizin, nur eben jetzt auf eine Person konzentriert.

Dass es den menschlichen Akteuren dabei in aller Regel an Expertise fehlt, hängt nicht nur damit zusammen, dass man schlecht mehrere zuvor getrennte, spezialisierte Aufgabenbereiche gleichermaßen kompetent wahrnehmen kann. Die fehlende Expertise hat auch damit zu tun, dass Typographie nicht an Schulen (und auch nicht an Universitäten) standardmäßig gelehrt und gelernt wird. Diese Institutionen des Bildungssystems sind in ihrem aktuellen Zustand tiefgreifend durch den Buchdruck geprägt und deren Akteure gehen bisher weitgehend davon aus, dass die Black Box Buchdruck in ihrer post-1900-Struktur intakt ist, dass es also keine Notwendigkeit gibt, einmal zu schauen, was an Tätigkeiten und Techniken notwendig ist, um Druckerzeugnisse zu produzieren, weil das ja die Black Box tut.

Aus kulturkonservativer Perspektive ließe sich dies beklagen; es könnte allerdings sein, dass das Bildungssystem in seiner Trägheit dann doch auf dem richtigen Weg ist. Sollte es nämlich beim Textverarbeiten irgendwann soweit sein, dass nicht mehr die bedruckte Seite das Modell abgibt; sollte es also so sein, dass die graphische Gestaltung einer Buchseite nicht mehr relevant ist, dann wird das typographische Wissen auch tatsächlich nicht mehr gebraucht. Wenn nämlich die textverarbeitende Nutzung des Computer darauf gerichtet ist „Texte [...] zum Gegenstand eines erleichterten Gebrauchs zu machen, sie einer möglichst mühelosen Manipulierbarkeit auf den verschiedenen Ebenen ihrer Ausführung (Buchstaben, Wörter, Sätze, Absätze, Abschnitte) zu unterwerfen“, ${ }^{20}$ dann träte an die Stelle der bedruckten (Buch-)Seite ein Textstrom, der sich verschieden materialisieren kann und sich nicht mehr auf der Fläche der (Buch-)Seite bändigen lässt. Das soll nicht heißen, dass schon morgen nur noch Textströme und keine Bücher mehr produziert werden, aber die kulturellen Energien werden sich verschieben (und tun dies ja auch schon seit geraumer Zeit durch die nicht mehr materialgebundenen Aspekte der Digitalisierung, des Internets, der Hyperlinks usw.). ${ }^{21}$

\section{Buch und Text}

Es hat gedauert, bis man vergessen hat, dass man den Druck und Bücher braucht, um Wissen zu produzieren und zu verbreiten. „Erst im Verlauf des 19. Jahrhunderts“, schreibt Michael Giesecke, „wurden die speziellen Medien und Informationsverarbeitungsprogramme, die zur Produktion des Wissens gebraucht wurden, so

20 Heilmann 2015, 586.

21 Man betrachte auch neuere digitale Literatur (vgl. etwa Bajohr 2016). 
selbstverständlich, so ,natürlich', dass man sie nicht weiter beachtete“. ${ }^{22}$ Man hatte irgendwann vergessen, dass das Medium relevant ist - und es wird bekanntlich dauern, bis dieses Wissen von Leuten reaktiviert wird, die sich insbesondere auch mit dem Buchdruck und der frühen Druckkultur beschäftigten. ${ }^{23}$

Es ist - zumal für einen Germanisten und also heute auch Literaturwissenschaftler - keineswegs unwichtig, dass die mediale Bindung von Wissen gerade im 19. Jahrhundert vergessen wurde, denn das 19. Jahrhundert sieht nicht nur die Etablierung des Dispositivs ,Literatur (im Anschluss an das, was man ,Weimarer Klassik‘ genannt hat), sondern auch die Etablierung der Germanistik als universitäre Disziplin. Beides, die Literatur und die Germanistik, fußen fest in der zeitgenössischen Buchkultur, die für normal und selbstverständlich gehalten wird. Das ist ein Grund, warum die Germanisten des 19. Jahrhunderts so viel Aufwand getrieben haben, um die Texte der mittelalterlichen Handschriftenkultur möglichst störungsfrei in die Druckkultur zu überführen. Die spezifische Form der Editionsphilologie, die gemeinhin mit dem Namen ,Karl Lachmann` aufgerufen wird, ist denn auch der brillante und ziemlich erfolgreiche Versuch, mittelalterliche Dichter zu Autoren zu machen - und mittelalterliche Handschriften zu Literatur.

Allem Anschein nach richtet sich die mediale Situation im 21. Jahrhundert neu aus, weg vom unhinterfragten Leitmedium Buch, hin zu einem „multimedialen Netzwerk“ ohne Prämierung eines Einzelmediums. ${ }^{24}$ Das allerdings bedeutet, dass die etablierte Vorstellung von Textualität als qua Druck stabilisiertes, standardisiertes und von vielen mittels identisch reproduzierter Bücher wahrnehmbares Wissen an ein Ende gelangt. Die inkorporierte Black Box Buchdruck des posttypographischen Zeitalters verfügt über flexible Wege des In- und Output, produziert keine Bücher, sondern flexible Textströme, die sich nicht qua Buchdeckel zu anderen Textartefakten hin abschließen, sondern sich an ein vielfach miteinander verbundenes Textnetzwerk anschließen. Der Textbegriff ist in diesem Zusammenhang entscheidend, weil er - zumindest in den Literaturwissenschaften - spätestens seit den 1960er Jahren dazu beigetragen hat, sich von Begrenzungsbegriffen wie insbesondere ,Autor und ,Werk‘ abzuwenden. Diese „Abkehr setzte dem vornehmlich sinnorientierten, autorzentrierten und statischen Konzept des ,Werkes“ mit dem Konzept des ,Textes` ein strukturorientiertes, eher deskriptives und dynamisches Modell entgegen“ “ ${ }^{25}$

Was freilich diesen frei fließenden Textströmen noch im Weg steht, sind die allgegenwärtigen WYSIWYG-Programme. Auch wenn diese Programme viel von der Schreibmaschine gelernt haben, sind sie doch zugleich als Teil der computerisierten Arbeitsumgebung auch die späten Nachfolger der Offizin und sie stellen viele der Werkzeuge bereit, die es braucht, um ein Buch zu produzieren, gerade dann, wenn

22 Giesecke 2005, 16.

23 Ong 1958; McLuhan 1962.

24 Giesecke 2005, 19.

25 Kammer/Lüdeke 2005, 13. 
dieses Buch (zumindest zunächst) ,nur` ein digitales Artefakt ist. Überhaupt bewegen sich die meisten dieser Programme mit ihrer Materialitätssimulation zwischen Schreibmaschine und Offizin, zwischen einem eingespannten, weißen Blatt Papier und dem Setzkasten, aus dem man sich bedienen kann. Zwar mag es sein, dass die wenigsten in der Lage sind, mit Hilfe etwa von Microsofts Word typographisch ansprechende Bücher zu fabrizieren - aber diese Black Box ließe sich bei Interesse leicht öffnen. Anstatt mit der Tastatur Text einzugeben und diesen mit wenigen Befehlen gemäß dem persönlichen Geschmack zu manipulieren, ließe sich ja durchaus ein typographisch anspruchsvolles Dokument generieren. Die technischen Möglichkeiten sind vorhanden, sie müssten nur genutzt werden.

Zugleich aber legt die Software gerade die weniger informierten NutzerInnen auf die Matrix des Buchdrucks fest: Es geht, so suggeriert das Programm in der Standardvoreinstellung, um die Gestaltung von (Buch-)Seiten. Solange nun aber die Matrix des Buchdrucks allgemeine Grundlage der Textverarbeitung ist, bleiben die Textströme zwar digital manipulierbar und haben also Potential, aber sie bleiben typographisch eingehegt. So gesehen lässt sich auf jeden Fall kaum sagen, dass das sogenannte Desktop Publishing der Untergang des Buchdrucks und der Buchkultur sei; eher im Gegenteil. Die Entgrenzung des Textes und die digitalen Textströme werden, im Fall des Desktop Publishing, durch traditionelle Elemente des Buchdrucks eingehegt und begrenzt. Insofern ist Word quasi die letzte Barriere, die die Textströme noch nach dem Modell des Gedruckten kanalisiert.

In Form von Computercode und Algorithmen werden sich die Textströme weiter verselbstständigen und unkontrollierbarer werden und vor allem für an Typographie gewohnte Akteure auch unlesbarer. Und während Gutenberg noch sein Labor und seine Werkstatt durch die Wahl des Ortes den Blicken Neugieriger entziehen konnte, leitet das Cloud Computing die Textströme in verschiedenste Richtungen, zu verschiedenen Geräten und vervielfältigt somit den Ort des Textes. Die Macht über den Zugang verschiebt sich dann hin zu Zugriffsrechten und im Vergleich zu Gutenbergs Labor und Werkstatt verschiebt sich die Black Box also von der Hardware hin zur Softwareseite.

\section{Fazit}

Über die Entwicklung vom Druck mit beweglichen Lettern ist viel geschrieben und nachgedacht worden; und auch an Reflexion über den aktuellen Stand des Schreibens und Publizierens fehlt es nicht. Wer sich in diese Diskurse - in beide Diskurse begibt, hat es nicht nur mit historischer Forschung, sondern mit ganzen Feuilletons, mit Sachbüchern, Verlautbarungen und zahllosen Blogeinträgen zu tun. Die Transformationslinien, die hier nachzuzeichnen waren, beruhen deshalb auf einer bescheidenen Versuchsanordnung. Was passiert, wenn man sich dem Druck mit 
beweglichen Lettern, dem routinisierten Buchdruck und schließlich der Situation des frühen 21. Jahrhunderts mit dem Konzept der Black Box nähert? Zu zeigen war, erstens, dass der frühe Druck mit beweglichen Lettern in zweifacher Hinsicht eine Black Box ist, weil nämlich einerseits die Technologie so gut es geht geheim gehalten wird und weil andererseits das Endprodukt anfänglich nach Kräften seine Herkunft aus der Maschine verbirgt. Zweitens wurde einmal mehr festgehalten, dass durch Standardisierung und Technisierung seit dem 18. und verstärkt seit dem späten 19. Jahrhundert das Medium (Buch-)Druck ,natürlich` wird, so dass man vergisst, dass das Medium relevant ist. Drittens schließlich lässt sich mit Blick auf das frühe 21. Jahrhundert diagnostizieren, dass die Black Box Buchdruck zur Textverarbeitung wird, zu einem Maschine-Mensch-Arrangement, bei dem nicht mehr Papier-Seiten gestaltet, sondern Textströme produziert werden. Die verschiedenen Rollen, die sich in der Offizin herausgebildet haben und die dann spezialisiert und verteilt wurden, werden nun zunehmend inkorporiert und damit auch nivelliert. Wenn ich selbst Autor, Lektor, Drucker und Buchhändler bin, dann bin ich nichts davon; was ich dann bin, kann man vielleicht ,Textverarbeiter' nennen, ein Verarbeiter von Textströmen im Maschine-Mensch-Arrangement. Das einzige, was uns vor den Fluten des Textes gegenwärtig noch zurückhält, sind die WYSIWYG-Programme. Pointiert gesagt: Microsofts Word ist die letzte Bastion der Druckkultur, weil diese als Matrix tief in der Software und deren Benutzerschnittstelle integriert ist.

\section{Literaturverzeichnis}

Bajohr, Hannes (Hg.) (2016), Code und Konzept. Literatur und das Digitale (Generator), Berlin. Cerquiglini, Bernard (1989), Éloge de la variante. Histoire critique de la philologie, Paris.

Eisenstein, Elizabeth L. (2011), Divine art, infernal machine. The reception of printing in the west from first impressions to the sense of an ending (material texts), Philadelphia/Oxford.

Giesecke, Michael (2005), „Auf der Suche nach posttypographischen Bildungsidealen“, in: Zeitschrift für Pädagogik 51, 14-29.

Giesecke, Michael (2017 [1991]), Der Buchdruck in der frühen Neuzeit. Eine historische Fallstudie über die Durchsetzung neuer Informations- und Kommunikationstechnologien (auch als suhrkamp taschenbuch wissenschaft 1357), Frankfurt a. M.

Hanebutt-Benz, Eva-Maria (2000), „Gutenbergs Erfindungen. Die technischen Aspekte des Druckens mit vielfachen Lettern auf der Buchdruckerpresse“, in: Stadt Mainz (Hg.), Gutenberg. aventur und kunst. Vom Geheimunternehmen zur ersten Medienrevolution. Katalog zur Ausstellung der Stadt Mainz anlässlich des 600. Geburtstages von Johannes Gutenberg, Mainz, 158-189.

Heilmann, Till A. (2012), Textverarbeitung. Eine Mediengeschichte des Computers als Schreibmaschine, Bielefeld.

Heilmann, Till A. (2015), „Textverarbeiten“, in: Heiko Christians, Matthias Bickenbach u. Nikolaus Wegmann (Hgg.), Historisches Wörterbuch des Mediengebrauchs, Köln/Weimar/Wien, 585-595.

Kammer, Stephan/Lüdeke, Roger (2005), „Einleitung“, in: Stephan Kammer u. Roger Lüdeke (Hgg.), Texte zur Theorie des Textes, Stuttgart, 9-21. 
Kittler, Friedrich A. ( ${ }^{4} 2003$ [1985]), Aufschreibesysteme $1800 \cdot 1900$, München.

McLuhan, Marshall (1962), The Gutenberg galaxy. The making of typographic man, Toronto.

Ong, Walter J. (1958), Ramus, method, and the decay of dialogue. From the art of discourse to the art of reason, Harvard.

Schorbach, Karl (1900), „Die urkundlichen Nachrichten über Johann Gutenberg“, in: Otto Hartwig (Hg.), Festschrift zum fünfhundertjährigen Geburtstage von Johann Gutenberg (Beihefte zum Centralblatt für Bibliothekswesen 8/23), Leipzig, 163-319.

Wagner, Sabina (2000), „Bekannter Unbekannter - Johannes Gutenberg“, in: Stadt Mainz (Hg.), Gutenberg. aventur und kunst. Vom Geheimunternehmen zur ersten Medienrevolution. Katalog zur Ausstellung der Stadt Mainz anlässlich des 600. Geburtstages von Johannes Gutenberg, Mainz, 114-143.

Zinn, Karl Georg (1989), Kanonen und Pest. Über die Ursprünge der Neuzeit im 14. und 15. Jahrhundert, Opladen.

\section{Bildnachweis}

Abb. 1: Public Domain, WikiCommons, „Französische Druckerwerkstatt zu Beginn des 16. Jahrhunderts“. (Quelle: Chants royaux sur la Conception, couronnés au puy de Rouen de 1519 à 1528 (fol. 29v), édition XVlème siècle. Bibliothèque Nationale de France, Département des manuscrits, Paris). 
